\title{
Construction of Dynamic Risk Maps for Large Metropolitan Areas
}

\author{
Evgeny S. Guryev ${ }^{1,2}$ \\ 1.Civil Engineering Institute, Ural State Federal University, Studencheskaya 54-A, Yekaterinburg 620049, Russia \\ 2.Science \& Engineering Centre, Ural Branch, Russian Academy of Sciences, Studencheskaya 54-A, Yekaterinburg \\ 620049, Russia \\ E-mail:sec@wekt.ru \\ Lyudmila V. Poluyan ${ }^{1,2}$ \\ 1.Civil Engineering Institute, Ural State Federal University, Studencheskaya 54-A, Yekaterinburg 620049, Russia \\ 2.Science \& Engineering Centre, Ural Branch, Russian Academy of Sciences, Studencheskaya 54-A, Yekaterinburg \\ 620049, Russia \\ E-mail:Poluyan@wekt.ru \\ Sviatoslav A. Timashev ${ }^{1,2}$ \\ 1.Civil Engineering Institute, Ural State Federal University, Studencheskaya 54-A, Yekaterinburg 620049, Russia \\ 2.Science \& Engineering Centre, Ural Branch, Russian Academy of Sciences, Studencheskaya 54-A, Yekaterinburg \\ 620049, Russia \\ E-mail:timashevs@gmail.com
}

Received 13 December 2013

Accepted 20 April 2014

\begin{abstract}
The paper describes a methodology used for constructing dynamic risk map for a virtual large «Russian GothamRG» city. The risk map is the core of the safety passport (SP) for the city, as required by the Russian EMERCOM and the Russian State Agency for Industrial Safety. The SP contains extensive risk analysis of all potential dangerous objects located within the boundaries of the city. It analyzes the natural and technological threats/disasters that may occur in the city.
\end{abstract}

Keywords: Disasters risk maps, large metropolitan areas

\section{Introduction}

The methodology of constructing dynamic risk maps (DRMs) for large metropolitan areas (LMAs) consists of following steps ${ }^{1,2}$ :

- Assessment of the potential territorial risk (PTR);

- Assessment of social risk (SR);

- Assessment of risk due to catastrophes and incidents when transporting HAZMATs by railways, on highways or by water;

- Assessments of risk of high and low pressure gas pipelines;
- Assessments of risk associated with hot/cold water supply systems and residential buildings fires;

- Assessment of risk due to natural hazards;

- Construction of social and property loss risk diagrams;

- Constructing risk fields on the digital map of the LMA (in our case - the RG-City);

- Update the risk fields every time some new data on risk comes to life or a new disaster scenario is considered.

Proceed to brief description of each of the above bullets. A full group of disaster scenarios is defined and the distribution of the probability of each scenario 
assessed, as well as their consequences. The conducted risk analysis shows that main sources of risk for the metropolitan area of the RG-City are railroads, highways, airport, gas stations, meat processing plant and some facilities that could release chlorine and/or ammonia. As the result, a digital map of the LMA showing the levels of risk (from $10^{-4}$ to $10^{-11}$ ) is constructed.

The dynamic risk map is a map which is updated every time some new data on risk comes to life. The main goal of such maps is to serve as an early diagnostics tool for decision making persons (DMPs) which are responsible for the well being of the population of the territory studied. The means that are needed for risk mitigation/reduction are then assessed in a timely manner.

\section{Potential Territorial Risk (PTR)}

PTR is a complex conditional measure of risk, which characterizes a potential dangerous object (PDO) or a territory. PTR is a 3D distribution of the conditional probability (frequency) of the possible realization of a negative influence of a specific level. When modeling dangerous technogenic processes associated with discharging harmful substances according to following scheme: "incident-impact process-realization of the impact”, the assessment of the PTR for an arbitrary point $(x, y)$ can be made using the following formula:

$$
R_{p t}(x, y)=\sum_{i, j} P\left(A_{i}\right) \cdot P_{i, j}(x, y) \cdot P\left(L_{j}\right)
$$

where $R_{p t}(x, y)$ is the potential risk, $P\left(A_{i}\right)$ is the probability of an incident according to the $i$-th scenario, $i=1, \ldots, I,\{I\}$ is a full group of events (FGE); $P_{i, j}(x, y)$ is the probability of manifestation of the $j$-th type impact in point for the $i$-th scenario of discharge, $j=1,2 . ., S,\{S\}$ is the FGE; $P\left(L_{j}\right)$ is the probability of a lethal (or any other) outcome during manifestation of the $j$-th type of impact (influence).

The conditional PTR (CPTR), by definition, is describing the potential of the maximal possible risk for concrete influenced objects, which are located in a given point $(x, y)$ of the territory in consideration. This measure of risk is conditional because it does not depend on:

- the very fact that the recipient is at the given point (it is assumed that the recipient is at the given point with probability of 1);
- the probability of the initiating event (it is assumed that $P\left(A_{i}\right)=1.0$ ).

Hence, the CPTR does not depend on whether the $\mathrm{PDO}$ is in a remote or urban environment, and becomes territorial conditional individual risk (TCIR). TCIR is equal to the probability (frequency) of affecting a human being (according to this or that type of hazard) due to development of a realization of a given type of impact in a point of the territory with given coordinates. In practice it is important to know the distribution of the potential risk for different sources of risk and specific scenarios of catastrophes. TCIR is therefore an intermediate measure of risk, which is used to assess the individual and the social risks.

\section{Definition of Social Risk}

SR characterizes the scale of possible incidents and is defined by the so called $\mathrm{F} / \mathrm{N}$ diagram (function). Depending on the purpose of the analysis $\mathrm{N}$ can be considered as the total number of injured people, number of fatalities or some other parameter that describes the seriousness of the catastrophe. Knowledge of the CPTR and of the distribution of the population across the studied territory allows getting quantitative assessment of the SR for the residents of a LMA.

The SR parameters are defined through the frequency of occurrence $(\lambda, 1 / \mathrm{yr})$ and the probabilistic zone of destruction $[P(x, y)]$ for each catastrophe scenario, when accounting for the distribution of the recipients $N(x, y)$ over the considered territory. The number of injured people $\left(N_{s c}, j\right)$ during a specific $i$-th scenario of an incident is defined by formula:

$$
N_{s c, j}=\int_{S} n(x, y) \cdot R_{I}(x, y) d s
$$

where $\lambda_{I}$ is the frequency of this event.

After calculating the number of injured people for the whole spectrum of possible scenarios $(j=1,2, \ldots, J)$ it is possible to draw the $\mathrm{F} / \mathrm{N}$ diagram, by summing up all the frequencies of incidents for which the number of injured is more than some preassigned value. Hence, the criteria of acceptable level of risk will be not a number, but a curve, constructed for different incident scenarios. Currently the generally accepted approach to defining the acceptability of risk is using two $\mathrm{F} / \mathrm{N}$ curves in logarithmic coordinates - one for acceptable risk and the other for unacceptable risk. The area between them is the grey zone where the level of risk is intermediate. The problem of decreasing this kind of risk is solved by 
accounting for the specificity of the industry, local aspects, and by coordinating with local bodies of self government and supervision. As a variable $\mathrm{T}$ in this case it is possible to take the economic (G) and/or ecological (E) damage.

\section{Constructing the Risk Fields on the Digital Map of a LMA}

The above results permit constructing integral and local risk fields. These fields are the most valuable forms of visualization first and foremost because they permit synthesizing a large number of nonhomogeneous data in a unique format which allows the decision making persons (DMPs) to easily understand the situation at hand. For constructing risk fields following data are used:

- A listing of the PDOs, described as a full group of $\mathrm{N}$ sources of hazard for the LMA in consideration;

- $\quad M$ types of possible incidents for each PDO from the listing, specifics of their occurrence and development;

- $\quad$ Results of drawing the CTPR fields;

- Probabilities of manifestation of the negative potential of these incidents $\lambda_{j}, j=\overline{1, M}$.

First local risk maps for each PDO are developed. Risk maps are constructed for each type of incident and each scenario that can physically occur in the considered PDO, with a concrete attachment to the source of danger. In this case the formula for calculating the total risk for a given PDO in each geo-cell $R_{\Sigma}(i, j)$ takes the form

$$
R_{\Sigma}(i, j)=\sum_{j=1}^{M} \lambda_{j} R_{I}(i, j)=\sum_{j=1}^{M} \lambda_{j} \cdot R_{I}(i, j)
$$

In this formula summation of risks is justified by the fact that all the incident scenarios and hence, the destruction zones, are independent.

As a result of these efforts a map $R_{I \Sigma}(i, j)$ of individual risk is formed, which characterizes the integral probability of this or that destructive factor, on the condition that the recipient with probability of 1 is in the given point of space during occurrence of the catastrophe. Having all the local maps they could be synthesized. Conduct summation of all the fields of potential danger for each PDO, taking into account their actual position on the LMA's map:

$$
R_{\Sigma}^{t}(i, j)=\sum_{k=1}^{N} R_{I \Sigma, k}(i, j)
$$

Here again it is justified to summate risks due to the mutual independence of the incidents in the PDO.

After constructing the integral risk field it is possible to assess the influence of the incidents/catastrophes on one object on the possibility of an incident in an adjacent PDO. This is especially important to take into account for scenarios which involve fires and explosions, because in these cases it is highly probable that cascade "domino" type accidents will develop, which, as a rule, lead to maximal economic and social damages.

\section{Assessment of Risk Due to Catastrophes and Incidents when Transporting Hazmats by Railways}

When analyzing possible scenarios two scenarios are picked for consideration: the most dangerous and the most probable. As the most dangerous scenario an incident on the railway was considered which involves destruction of several railroad tanks containing oil products. According to Russian State rule it is necessary to consider the case when half of all the oil carrying tanks are losing their contents. As a most probable scenario an oil spill from a single railway tank (120 cub.m) was considered. These scenarios can be comprised of following sub-scenarios (SS):

a. Oil products spill

SS 1.1 - oil spill from 25 tanks 80 cub. meters each $\left(2000 \mathrm{~m}^{3}\right)$; SS 1.2 - oil spill from a single tank $\left(120 \mathrm{~m}^{3}\right)$. Consequences of such incidents are: atmosphere pollution by oil products evaporation, intoxication of people. Possible secondary types of accidents could be oil spill fire, toxic injury of people, and creation of a fuel-air mixture cloud (FAMC) followed with its explosion.

b. Forming of a burning oil spill

SS 2.1 - oil spill from 25 tanks 80 cub. meters each $\left(2000 \mathrm{~m}^{3}\right)$ followed by its ignition; SS 2.2 - oil spill from a single tank $\left(120 \mathrm{~m}^{3}\right)$ followed by ignition of the spill. Dangerous factors which influence people and assets are flame and sparks, elevated temperature of the environment, toxic products from the fire and thermal discomposure of the materiel, smoke, decreased concentration of oxygen in the air. Possible secondary type incidents in this case are: fire ball and explosion, ignition of the technological equipment and transportation means, buildings and structures. 
c. Forming of a FAMC followed by a fire ball

SS 3.1- oil spill from 25 tanks 80 cub. meters each $\left(2000 \mathrm{~m}^{3}\right)$ followed by forming a fire ball; SS 3.2 - oil spill from a single tank $\left(120 \mathrm{~m}^{3}\right)$ followed by ignition of a FAMC. Consequences of such development of the incident are atmosphere pollution by products of burning oil, intoxication and thermal injury of people. Possible secondary incidents include: explosion, ignition of the technological equipment and transportation means, buildings and structures.

d. Forming of a FAMC followed by its explosion (creation of an air blast wave)

SS 4.1 - oil spill from 25 tanks 80 cub. meters each $\left(2000 \mathrm{~m}^{3}\right)$ followed by the FAMC explosion; C 4.2 - oil spill from a single tank $\left(120 \mathrm{~m}^{3}\right)$ followed by the FAMC explosion. In order to assess the most dangerous case the oil product considered in scenarios SS 4.1 - SS 4.2 was benzene. In risk calculations the surrounding air temperature was taken as $20^{\circ} \mathrm{C}$. The consequences of this kind of catastrophe development could be: destruction of equipment, air pollution, destruction of flora, and intoxication of people, injuries of people by the blast wave and by equipment projectiles. Due to the blast a fire ball could be created. Possible secondary type incidents in this case are: fire ball, ignition and destruction of the technological equipment and transportation means, buildings and structures. Collective risk for the most probable incident is $2.16 \cdot 10^{-}$

3 . Collective risk for the most dangerous incident is $6.2 \cdot 10^{-4}$

In this study chlorine spill on railways was also considered and its consequences evaluated. The typical scenario involves three tanks on a railway station, each containing 60 tons of chlorine. The frequency of this type of incident is $3.2 \cdot 10^{-6}$. The intoxication radii is 2.5 $\mathrm{km}$, the radii of lethal intoxication is $0.87 \mathrm{~km}$. The average number of fatalities, at average population density for the RG-City being 0.0022 residents $/ \mathrm{m}^{2}$ is 412 people. Collective risk is equal to $1.28 \cdot 10^{-3}$.

\section{Assessments of Risk when Transporting Hazmats Along Highways}

The incident frequency during highway transportation for the case when 4 containers with chlorine are transported is equal to $3.8 \cdot 10^{-4}$. It is assumed that each container contains $850 \mathrm{~kg}$ of chlorine. Assume that the average time of transportation of chlorine inside the LMA is not more than 2 hours. In this case the radii of intoxication will be $2.5 \mathrm{~km}$, the radii of lethal intoxication is $0.86 \mathrm{~km}$. The average number of fatalities, at average population density for the RG-City being 0.0022 citizens $/ \mathrm{m}^{2}$ is equal to 412 people. Collective risk is equal to $1.4210^{-2}$.

\section{Assessment of Risk for High (900kpa) and Low (50kpa) Pressure Gas Pipelines}

The average frequency of loss of containment for small diameter trunk gas pipeline per $\mathrm{km}$ yr is $2.8 \cdot 10^{-4}$. The probability of gas cloud explosion is 0.0118 . Calculations were conducted taking into account possible deaths of people during destruction of buildings and on the streets of LMA. For high pressure gas pipelines individual risk IR is $3.91 \cdot 10^{-9}$. For low pressure gas pipelines IR is equal to $1.5 \cdot 10^{-9}$. The total individual risk when trunk gas pipelines operate is $5.4 \cdot 10^{-9}$

\section{Assessments of Risk Associated with Hot/Cold Water Supply Systems and Residential Buildings Fires}

All calculations are based on the statistics provided by the local ENERCOM based on statistics gathered during the time interval years (2002 - 2005). Results of this analysis are not shown in this paper.

\section{Assessment of Risks due to Natural Hazards}

In the RG-City the average duration of a thunderstorm is 90 minutes, the average number of storms per year is 25.6. The average number of thunderbolts per sq.km year as given by the Russian Code GOST 12.1.004-91 is equal to 3 (thunderbolts $\mathrm{km}^{2} / \mathrm{yr}$ ). According to this code the probability $Q_{i}(t)$ that a thunderbolt will directly strike a building is found as

$$
Q_{1}(t)=1-e^{-N_{t s} \tau_{d}}
$$

where $N_{t s}$ is the number of direct strikes of a thunderbolt of an infrastructure object, per year; $\tau_{d}$ is the duration of local observations of this phenomenon, years.

For rectangular objects

$$
N_{t s}=(S+6 H) \cdot(L+6 H) \cdot N_{t s} \cdot 10^{-6}
$$

Assume the average height of the RG-City citizen is equal to $1.5 \mathrm{~m}$, the probability of a person being 
outdoors during a storm is not more than $1.1 \cdot 10^{-3}$. Then the individual risk of being killed by a thunderbolt is not more than $2.6 \cdot 10^{-7}$.

The average number of days when the outside temperature in the RG-City is below $-25^{\circ} \mathrm{C}$ (according to meteorological data) is $\geq 8$. According to expert assessments the probability that a person would die due to hypothermia is $1.1 \cdot 10^{-7}\left(\right.$ days $\left.^{-1}\right)$. Then the individual risk of death due to hypothermia is not more than $8.2 \cdot 10^{-7}$. Loss of people due to earthquakes and hurricanes in the vicinity of the RG-City was never registered. The total individual risk of life loss due to natural hazards in the RG-City is $1.04 \cdot 10^{-6}$.

\section{Construction of Social and Property Loss Diagrams}

All the above allowed constructing F/N social diagrams for employees of companies that are located in the LMA, its residents and the total diagram (see Figs. 1-3) and a generalized F/G diagram for the city (see Fig. 4).

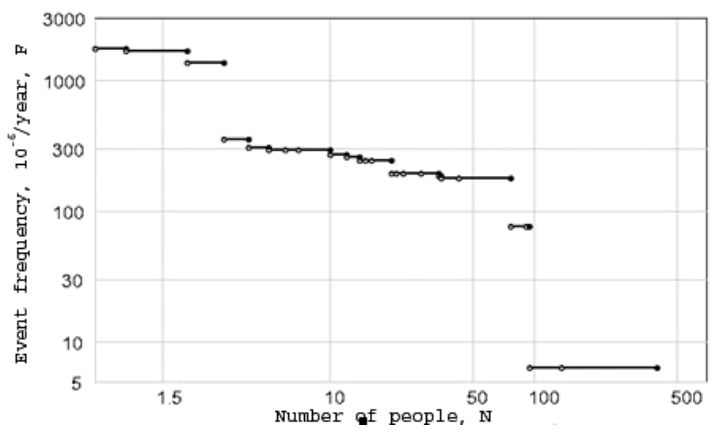

Fig. 1. F/N social risk diagram for companies' employees.

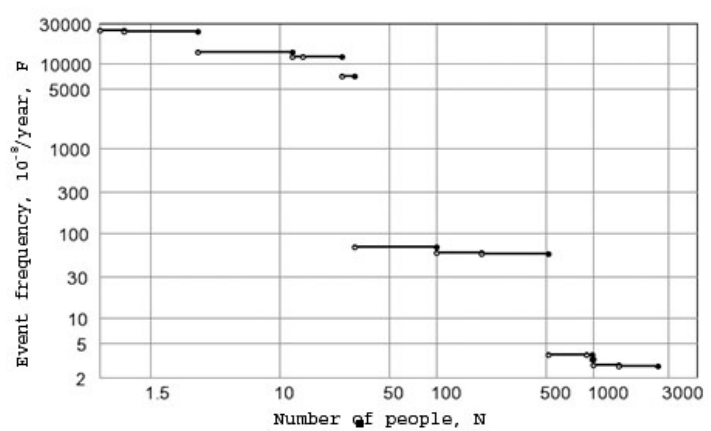

Fig. 2. F/N social risk diagram for the population.

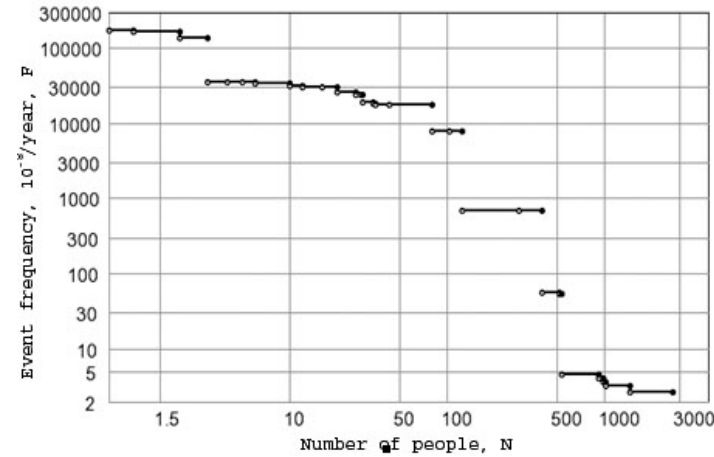

Fig. 3. Total social risk F/N diagram.

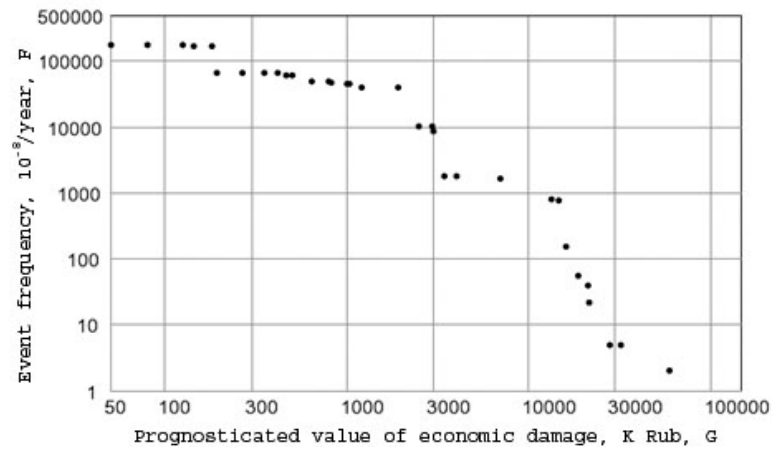

Fig. 4. F/G diagram.

\section{Conclusion}

The outlined methodology for constructing individual risk maps is an important and useful tool for providing insight into every decision made by the decision making persons that govern the LMA.

\section{References}

1. S. A. Timashev, Management of risk of interdependent systems of critical infrastructures and territories, in Proc. II All Russia Science and Technology Conf. Safety of Critical Infrastructures and Territories (Yekaterinburg, Russia, 2008), pp. 7-9.

2. S. A. Timashev, Risk and entropy based quantitative definition and solution of regional resilience and strategic preparedness, Ibid (2008), pp. 261-265. 